\title{
Solid-supported lipid multilayers under high
}

\section{hydrostatic pressure}

Benedikt Nowak ${ }^{a,+\dagger}$ Michael Paulus, ${ }^{\dagger}$ Julia Nase,${ }^{\dagger}$ Paul Salmen, ${ }^{\dagger}$ Patrick Degen, ${ }^{\ddagger}$ Florian J. Wirkert, ${ }^{\dagger}$ Veijo Honkimäki, ${ }^{\text {II }}$ and Metin $\operatorname{Tolan}^{\dagger}$

Fakultät Physik/DELTA, TU Dortmund, 44221 Dortmund, Germany, Physikalische Chemie II, TU Dortmund, 44221 Dortmund, Germany, and European Synchrotron Radiation Facility, F-38043 Grenoble, France

E-mail: benedikt.nowak@tu-dortmund.de

\section{Supporting Information}
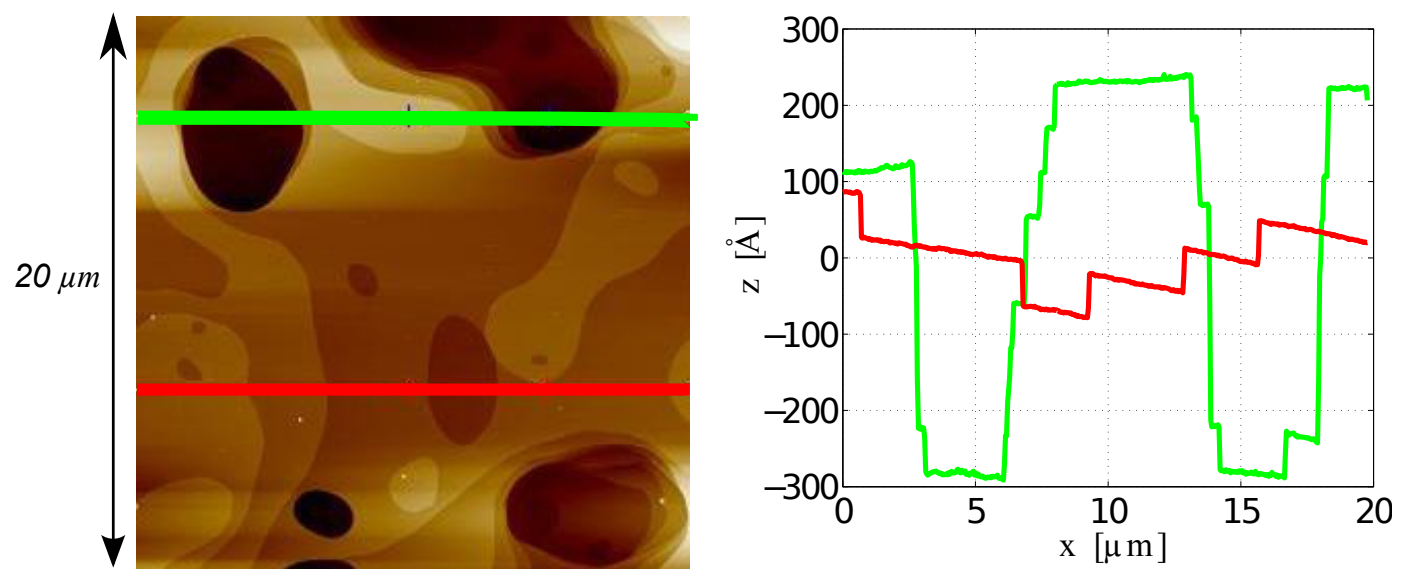

Figure S1: Left: AFM picture of a DMPC multilayer. Right: height profile along the lines drawn on the left side.

*To whom correspondence should be addressed

${ }^{\dagger}$ TU Dortmund, Fakultät Physik

†U Dortmund, Fakultät Chemie

${ }^{\mathrm{I}} \mathrm{ESRF}$ 


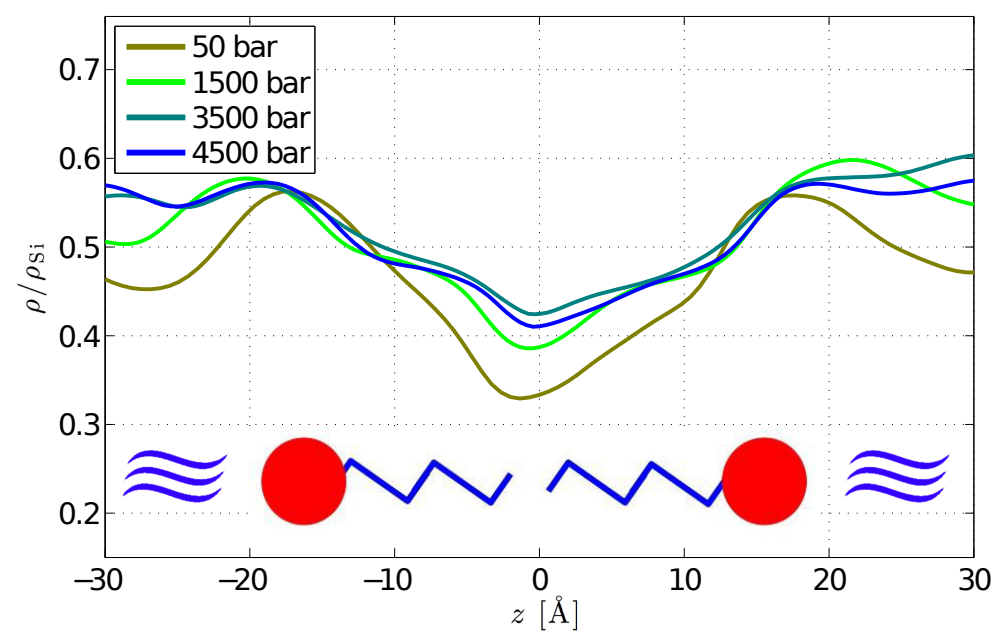

Figure S2: Zoom of a bilayer from the DMPC multilayer in figure 3. Curves for each pressure are shifted so that the region between the lipid tails is centered for better comparability.

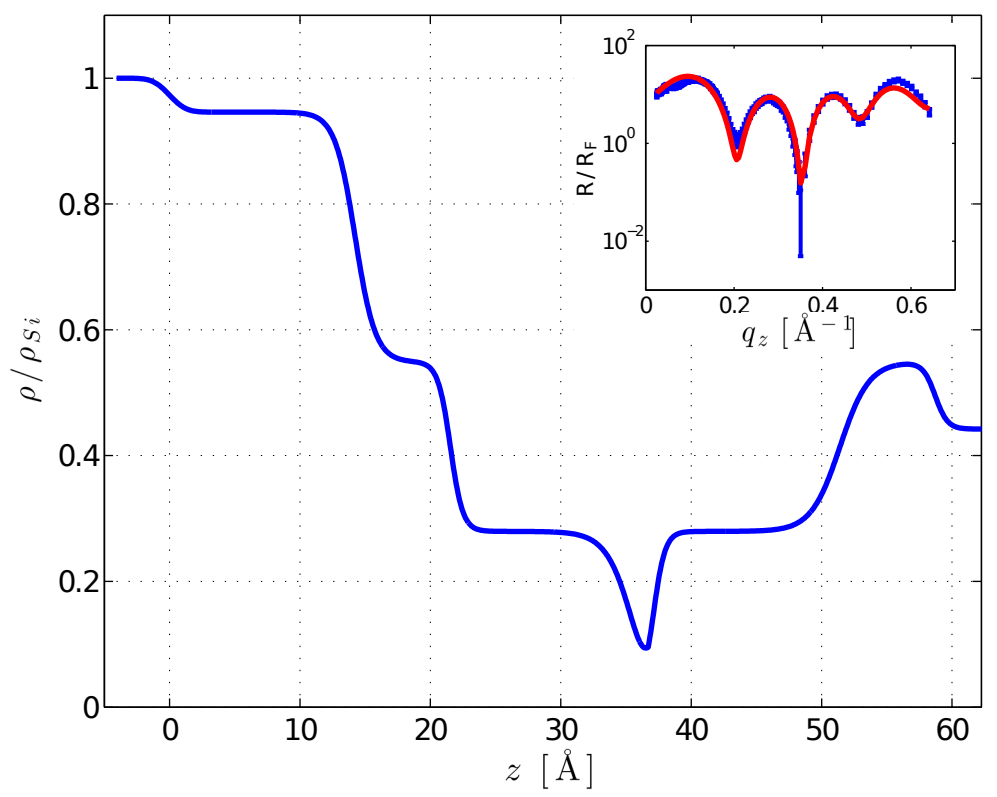

Figure S3: Electron density profile of the bilayer at 50 bar. Inset: Corresponding XRR data with fit. 